\title{
Case Report Capnocytophaga canimorsus: infection,
septicaemia, recovery and reconstruction
}

\author{
Stephanie Chiang-Mei Low and John Edward Greenwood
}

Correspondence

Stephanie Chiang-Mei Low

Burns Unit, Royal Adelaide Hospital, Adelaide, South Australia 5000, Australia

stephanie.low@health.sa.gov.au

Received 8 November 2007

Accepted 11 March 2008

\begin{abstract}
A case is presented of a life-threatening septicaemia and associated peripheral necrosing microembolic phenomenon, resulting from a dog lick to an insignificant burn wound. The isolated bacterium was Capnocytophaga canimorsus, a slow-growing Gram-negative bacillus commonly found in dog saliva. Any clinician seeing patients with a history of dog bite/saliva contact and progressive illness should consider this bacterium as a possible offender and take special care to elicit an accurate history, specifically including questions regarding animal contact.
\end{abstract}

\section{Introduction}

Although human bites have the greatest propensity for wound infection, because of the virulent and varied flora residing in the human mouth and the tendency for associated crush injury, dog bites are more common (Moore, 1997). A survey conducted by the South Australian Health Commission in 1991, estimated that up to 30000 people presented to South Australian hospitals annually as the result of dog attacks in Australia (South Australian Health Commission, 1991; Thompson, 1997). A second report produced in 1997 revealed that 6500 people are injured by dogs in Adelaide each year, 810 of whom seek hospital treatment (Thompson, 1997).

The most common pet per household in Australia is the dog and many people are exposed to dog saliva daily (Australian Bureau of Statistics, 1995). Many dog-bite victims regard their injuries as minor or insignificant and do not seek medical attention. Of all dog bite victims, a small proportion develop overwhelming septicaemia (life threatening), subsequent multi-organ failure and ultimately die. The genus Capnocytophaga is part of the family Flavobacteriaceae and is in the Flavobacterium-Cytophaga rRNA homology group (Mandell et al., 2005; Vandamme et al., 1996).

These organisms are found in the oral cavity of both humans and canine species (Mandell et al., 2005). Capnocytophaga species isolated from the human oral cavity were originally described by Prevot and colleagues in 1956 (Prevot et al., 1956; Mandell et al., 2005; Bobo \& Newton, 1976). In 1976 a previously undescribed Gramnegative rod was isolated from the blood and spinal fluid of a patient after a dog bite (Bobo \& Newton, 1976; Hore, 2001) and was denoted as dysgonic fermenter 2 (DF-2). In 1989, Brenner and colleagues (Brenner et al., 1989) proposed the name Capnocytophaga canimorsus for this species (canimorsus being derived from the Latin for 'dog bite') (Hore, 2001). Further investigation also revealed a second biochemically different group that were named
Capnocytophaga cynodegmi (cynodegmi being derived from the Greek for 'dog bite') (Hore, 2001). C. canimorsus is a Gram-negative filamentous, slow-growing, non-sporeforming facultative aerobe found in the oral cavity of canines (Hore, 2001). It is susceptible to penicillins, imipenem, erythromycin, vancomycin, clindamycin, third-generation cephalosporins, chloramphenicol, rifampicin, doxycycline and quinolones (Hore, 2001). However, it is resistant to aztreonam, trimethoprim-sulfamethoxazole and aminoglycosides (Hore, 2001). There have been a small number of case reports previously reporting human illness involving C. canimorsus.

The common presenting history is an immunocompromised patient who sustains a dog/cat bite. The incubation time for the bacterium ranges between 1 and 8 days (Hore, 2001). During this time the patient becomes increasingly unwell, with initial lethargy, tiredness, listlessness, and subsequent fevers, rigors and embolic events, including necrosis of the extremities. Multi-system organ failure follows (Hore, 2001). In these scenarios, infection is often fatal, with mortality rates in the order of $30 \%$ (Hore, 2001; Scully et al., 1999; Pers et al., 1996).

\section{Case report}

On June 242007 at 17.45, a previously fit and healthy 48year-old woman was admitted to Mildura Base Hospital, Mildura, Victoria, Australia, with abdominal pain. She described a $24 \mathrm{~h}$ history of epigastric pain, nausea, vomiting, diarrhoea, rigors and headaches. She omitted (as unimportant) the history of a hot water scald some 3-4 weeks prior to presentation resulting in a superficial dermal burn on the dorsum of her left foot (approximately $33 \mathrm{~mm}$ in diameter - much less than $1 \%$ of her body surface area). Nor did she realize the significance of her fox terrier puppy subsequently licking the burn area. The burn wound was not cleaned appropriately, nor antibiotic therapy sought. 
This was a significant piece of the history, since it reveals the source and cause of all the symptoms and signs.

On examination she was hypotensive, peripherally shut down and febrile. She maintained her own airway but was speaking in short sentences, and the attending clinician was unable to obtain a good oxygen saturation level by pulse oximetry due to poor peripheral perfusion. Her limbs were reportedly cold, white and mottled, with her face and torso developing a purple hue. Petechial haemorrhage was suspected for the mottling and central discoloration. Consistent with the poor peripheral perfusion, her feet demonstrated decreased sensation, but retained good movement. She underwent abdominal computed tomography, which showed an enlarged gallbladder with the possibility of cholelithiasis, but no free gas or significant abdominal free fluid. She was severely oliguric.

With this picture of septic shock, she received blind broadspectrum antibiotics after blood cultures were sent for laboratory analysis: $1 \mathrm{~g}$ ceftriaxone at $18.35,500 \mathrm{mg}$ metronidazole at 19.00 and $1 \mathrm{~g}$ meropenem at 20.20 . Antibiotics did not alter the progression of sepsis or cyanotic symptoms and at 20.00 nursing staff reported progressive cyanosis with increased mottling of her ear lobes. She was moved into a resuscitation cubicle and a review by senior medical staff was requested.

At 20.30 she was reviewed by the Royal Adelaide Hospital Medi-Flight Retrieval Team who had arrived to retrieve another patient. The team were extremely concerned about her and transferred her immediately to the Royal Adelaide Hospital, arriving shortly after midnight, on the morning of the $25^{\text {th }}$ June.

At the Royal Adelaide Hospital, investigations confirmed septic shock, with acidosis, leucopenia and patterns typical of disseminated intravascular coagulopathy. She was intubated. Echocardiography revealed a dilated inferior vena cava and right ventricle with subsequent right ventricular dysfunction. Inotropes were commenced (dobutamine and noradrenaline) to maintain central circulation and systolic pressure, and to support renal and cardiac function. A review of admission blood results revealed plummeting white cell counts, and coagulation and renal parameters dramatically abnormal: white cell count $2.43 \times 10^{9}$ cells $1^{-1}$

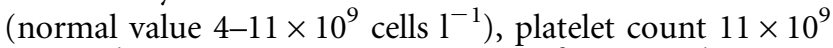
cells $1^{-1}$ (normal value $150-400 \times 10^{9}$ cells $1^{-1}$ ), international normalized ratio 4.7 (normal value 1), activated partial thromboplastin time $>200 \mathrm{~s}$ (normal value $25-35 \mathrm{~s}$ ), lactate $8.0 \mathrm{mmol} \mathrm{l}^{-1}$ (normal value $0-2.2 \mathrm{mmol} \mathrm{l}^{-1}$ ), creatinine $160 \mu \mathrm{mol} \mathrm{l}^{-1}$ (normal value $40-90 \mu \mathrm{mol} \mathrm{l}^{-1}$ ).

On admission to the intensive care unit (ICU), she was diagnosed with septic shock and multi-organ failure, including adult respiratory distress syndrome, acute renal failure, disseminated intravascular coagulopathy, cardiovascular failure and leucopenia, the cause being queried as meningococcal septicaemia. Over the following 3-4 weeks, her medical state improved, with her renal, respiratory, immune, cardiovascular function and coagulopathy returning to normal.

On July 122007 the Royal Adelaide Hospital Burns Team were asked to review her in the ICU. Her medical condition had improved, but she had areas of black eschar on the tips of her fingers, toes, nose and various other areas (including thighs and upper arms). On examination it was clear that these darkened areas represented dry necrosis and other non-escharified tissues were discoloured and non-viable. Salvage of the majority of these tissues was impossible.

It was only at this point that an organism was cultured from the patient's blood: the causative organism was $C$. canimorsus. Following research into this unusual organism, further inquiry was made as to how this particular patient could have been infected. The common sites for $C$. canimorsus growth were discovered, and it was only then that the patient realized the significance of her small burn wound and the dog lick.

The patient was taken to theatre over the following fortnight to examine the necrotic areas, debride non-viable tissue and dress wounds using non-adhesive and antibacterial dressings. Unfortunately debridement included removal of all fingers and all toes, at the metacarpophalangeal joint/metatarsophalangeal joint level, and both thumbs at interphalangeal joint level. A below knee amputation to her left lower limb and a forefoot amputation to her right lower limb were performed. At each debridement, as much viable tissue was preserved as was possible, without putting life and vital organs in danger.

After three operative debridement procedures to eradicate all necrotic tissues, it took a further three definitive operations to close her wounds. Definitive procedures included a left below-knee amputation, amputation of all digits, with split skin grafts to cover the distal tissue defects. We also undertook reconstruction of her right forefoot. A left radial forearm free flap was harvested and anastomosed to the anterior tibial artery on the right foot, with the skin paddle overlying the medial, distal, lateral and dorsal aspects of the forefoot. The minor residual defect $(3 \times 4 \mathrm{~cm})$ on the medial aspect of her right sole was filled with Integra dermal regeneration template, as was the defect created on her left forearm (from the free flap donor site). The Integra was dressed and left to integrate over the following 5 weeks, at which point the Integra sites were covered with sheet split skin graft sutured into place. The Integra filled the defect created by the harvesting of the radial forearm flap superbly and generated the best result possible.

\section{Discussion}

C. canimorsus is a common Gram-negative animal oral commensal bacterium, with a severe and often fatal course once sepsis becomes established. The common presentation of C. canimorsus reported in literature is a dog or cat bite in an immunocompromised patient. Immunocompromisation seems to be a common factor in the majority 
of C. canimorsus sepsis cases. Patients are often lymphoma or leukaemia sufferers who sustain a dog or cat bite, and 714 days later become progressively unwell. Early signs are lethargy, tiredness and listlessness, while the progression is to fevers, rigors, embolic events and eventual multi-system organ failure.

This case is unique in that the patient was not immunocompromised at the time of contact with the dog saliva. The other significant difference is that she did not sustain a bite from her dog, but merely a lick to a superficial burn, the only possible site of entry for the bacterium.

An important 'take away' message from this case report is that domestic pets carry potentially lethal bacteria, and infection even in immunocompetent individuals can result in loss of life or limb. The seriousness of this particular presentation mandates a general awareness of $C$. canimorsus. Careful history taking might elucidate contact, enabling recognition before such serious sequelae.

\section{References}

Australian Bureau of Statistics (1995). Special feature: household pets. In Australian Social Trends, 1995, 4102.0, culture and leisure. http://www.ausstats.abs.gov.au/ausstats/free.nsf/0/FFD0195188E1223 FCA25722500049552/\$File/41020_1995.pdf

Bobo, R. A. \& Newton, S. J. (1976). A previously undescribed Gramnegative bacillus causing septicaemia and meningitis. Am J Clin Pathol 65, 564-569.
Brenner, D. J., Hollis, D. G., Fanning, G. R. \& Weaver, R. E. (1989). Capnocytophaga canimorsus sp. nov. (formerly CDC group DF-2), a cause of septicemia following dog bite, and C. cynodegmi sp. nov., a cause of localized wound infection following dog bite. J Clin Microbiol 27, 231-235.

Hore, C. (2001). Important unusual infections in Australia: a critical care perspective. Crit Care Resusc 3, 262-272.

Mandell, G. L., Bennett, J. E. \& Dolin, R. (editors) (2005). Principles and Practice of Infectious Diseases, 6th edn, chapter 231, Capnocytophaga. Philadelphia, PA: Churchill Livingstone.

Moore, F. (1997). I've just been bitten by a dog. BMJ 314, 88-90.

Pers, C., Gahrn-Hansen, B. \& Frederiksen, W. (1996). Capnocytophaga canimorsus septicaemia in Denmark, 1982-1995: review of 39 cases. Clin Infect Dis 23, 71-75.

Prevot, A. R., Tardieux, P., Joubert, L. \& de Cadore, F. (1956). Studies on Fusiformis nucleatus (Korr) and its pathogenicity for humans and animals. Ann Inst Pasteur (Paris) 91, 787-798.

Scully, R. E., Mark, E. J., McNeely, W. F., Ebeling, S. H., Phillips, L. D. \& Ellender, S. M. (1999). Case records of the Massachusetts General Hospital. Weekly clinicopathological exercises. Case 17-1999. A 42year-old asplenic man with Gram-negative sepsis. N Engl J Med 340, 1819-1826.

South Australian Health Commission (1991). Dog attacks. Inj Surveill Monthly Bull 29, 1-2.

Thompson, P. G. (1997). The public health impact of dog attacks in a major Australian city. MJA 167, 129-132.

Vandamme, P., Vancanneyt, M., Van Belkum, A., Segers, P., Quint, W. G. V., Kersters, K., Paster, B. J. \& Dewhurst, F. E. (1996). Polyphasic analysis of strains of the genus Capnocytophaga and Centers for Disease Control group DF-3. Int J Syst Bacteriol 46, 782791. 